\title{
ADRENAL CARCINOMA: A REVIEW OF FOUR CASES
}

Background: Adrenal carcinoma is rare endocrine cancer. Survival time is short and primary treatment is surgery.

Methods: Four patients with histopathologically diagnosed as adrenal carcinoma between 2007 to 2013 were evaluated retrospectively.

Results: Two of the patients were female and others were male. Their ages were 39, 50, 55 and 61 respectively. All the patients were admitted to the hospital with abdominal pain. Tumor diameters of the cases were 20,18,5 and $11 \mathrm{~cm}$ respectively. Tumor was on left side in 3 patients and on the right side in 1 patient. Three patients had active hormone secreting tumors that was cortisol. One patient had hormone-inactive tumor. Two patients were stage-4, one patient was stage- 2 and one patient was stage-1 according to TNM classification. Two patients were treated with mitotane. Two of the patients died 14 and 15 months after the operation respectively. One patient (39 years old) is alive for 3 years after the operation. One patient is alive for 6 months after the operation and he is still taking the treatment of mitotane.

Conclusion: Adrenal carcinomas have bad prognosis. The patients have been diagnosed at late stages. The most important survival criteria is complete surgical resection. The age of the patient at diagnosis might be an another survival factor. Mitotane treatment might be used in selected patients. 